\title{
Follicle-stimulating hormone signal transduction: role of carbohydrate in aromatase induction in immature rat Sertoli cells
}

\author{
Vasantha Padmanabhan, Malur R. Sairam, Jeanne M. Hassing, Morton B. Brown, \\ Jane W. Ridings and Inese Z. Beitins \\ Department of Pediatrics, Division of Pediatric Endocrinology, Unitersity of Michigan, Ann Arbor, MI 48109-0718, U.S.A., \\ and Reproduction Research Laboratory, Clinical Research Institute of Montreal, Montreal, Quebec, Canada
}

(Received 6 January 1991; accepted 1 May 1991)

Key words: Follicle-stimulating hormone; Deglycosylation; Cyclic AMP response; Aromatase induction; Glycoprotein; Gonadotropin.

\section{Summary}

Receptor activated adenylate cyclase acts as a major transmembrane signalling system. It is widely accepted that upon binding to its receptor, follicle-stimulating hormone (FSH) activates the cAMP-dependent pathway which in turn mediates FSH-induced estradiol production in Sertoli cells. Studies utilizing several chemically derived variants of FSH have demonstrated that these variants bind to the FSH receptors with equal avidity but differ in their ability to activate cAMP-dependent pathways. Since cAMP is believed to be the second messenger responsible for FSH signal transduction, we tested two hypotheses: (1) that the effects of different oFSH variants on cAMP production and aromatase induction (as measured by estradiol production) would be in parallel; and (2) that deglycosylated ovine FSH (DG-oFSH) would antagonize the ability of intact oFSH to stimulate aromatase induction, similar to its reported antagonistic effect on cAMP production.

Immature rat (7- to 10-day-old) Sertoli cells were cultured and the effects of several different oFSH variants on cAMP production and/or aromatase induction were tested. The variants tested were native oFSH, DG-oFSH, asialo oFSH (AS-oFSH), a recombinant of intact $\mathrm{LH} \alpha$ and FSH $\beta(\alpha+\beta)$ and a recombinant of deglycosylated $\mathrm{LH} \alpha$ and intact $\mathrm{FSH} \beta(\mathrm{DG} \alpha+\beta)$. Both native oFSH and $\alpha+\beta$ recombinant at relatively large doses $(10 \mathrm{ng})$ elicited a significant increase in extracellular cAMP accumulation as well as total cAMP production. In contrast, DG-oFSH did not produce an increase in cAMP even at 10 -fold higher doses than native oFSH. Intracellular cAMP concentrations did not increase following stimulation with native oFSH, DG-oFSH or DG $\alpha+\beta$.

In contrast to the divergent effects of oFSH and DG-oFSH on cAMP production all variants of oFSH stimulated estradiol production from Sertoli cells albeit with varying potencies. The sensitivity (minimal

Address for correspondence: Dr. Vasantha Padmanabhan, Department of Pediatrics, Division of Pediatric Endocrinology, University of Michigan, Ann Arbor, MI 48109-0718, U.S.A.

Presented in part at the 21st Annual Meeting of the Society for the Study of Reproduction, Columbia, MO, U.S.A.

Supported by NIH grants HD 23812 (V.P.), -18515 (I.Z.B.) and MT 5475 of MRC of Canada (M.R.S.).
Abbrcviations: oFSII, native ovine follicle-stimulating hormone; DG-oFSH, deglycosylated ovine FSH; $\alpha$, luteinizing hormone ( $\mathrm{LH}) \alpha$ subunit; $\beta$, FSH $\beta$ subunit; DG $\alpha$, deglycosylated $\mathrm{LH} \alpha ; \mathrm{AS}-\mathrm{oFSH}$, asialo or desialylated ovine $\mathrm{FSH}$; cAMP, cyclic adenosine mono-phosphate; $\alpha+\beta$, recombinant of intact ovine $\mathrm{LH} \alpha$ and intact ovine $\mathrm{FSH} \beta ; \mathrm{DG} \alpha+\beta$, recombinant of deglycosylated ovine $\mathrm{LH} \alpha$ and intact ovine FSH $\beta$. 
effective dose) and $E D_{50}$ (dose at which half maximal response is achieved) of the estradiol $\left(E_{2}\right)$ response curve to increasing concentrations of native oFSH were $0.025 \pm 0.01$ and $0.33 \pm 0.05 \mathrm{ng}$, respectively. Asialo-oFSH (AS-oFSH) increased $\mathrm{E}_{2}$ production with a potency (comparative dose required for effect) similar to that of native oFSH. In contrast, there was a 10 -fold reduction in potency of $\mathrm{DG}$-oFSH (ED $\mathrm{ED}_{50}$ of $3.27 \pm 1.7 \mathrm{ng}$ ). The efficacy (maximum effect on aromatase induction) of native, DG and AS-oFSH were equipotent. Coincubation of DG-oFSH $(0.005-100 \mathrm{ng})$ with oFSH $(0.01-0.625 \mathrm{ng})$ demonstrated the inductive effects to be additive. In contrast to the reduced potency of $\mathrm{DG}-\mathrm{oFSH}, \mathrm{DG} \alpha+\beta$ recombinants $(0.156-160 \mathrm{ng})$ increased $E_{2}$ production with a potency similar to that of the native oFSH.

Our data suggest: (1) the effects of oFSH variants on cAMP production and aromatase induction are not always parallel; (2) DG-oFSH is a weak agonist and not an antagonist of oFSH-mediated aromatase induction; (3) the reduced potency of DG-oFSH must involve oligosaccharides internal to sialic acid; and (4) depleting the carbohydrate moiety on the $\alpha$ subunit alone may not be adequate to reduce the potency of oFSH to induce aromatase. Since DG-oFSH does not increase cAMP accumulation, but is able to induce aromatase, the FSH-mediated induction of aromatase may be transduced through other signal pathways. Alternatively, the maximal steroidogenic response may be induced by small, but undetectable, increases in intracellular cAMP concentrations or availability of segregated cAMP pools.

\section{Introduction}

Follicle-stimulating hormone (FSH) like other glycoprotein hormones is a heterodimer consisting of two non-covalently bound subunits, $\alpha$ and $\beta$. The $\alpha$ subunits of FSH, luteinizing hormone (LH), human chorionic gonadotropin (hCG) and thyrotropin (TSH) are identical, while the $\beta$ subunits are hormone-specific and confer biological activity (Sairam, 1989). Both the FSH $\alpha$ and $-\beta$ subunits contain two asparagine-linked oligosaccharides (Sairam, 1983). Human FSH oligosaccharides terminate with sialic acid, but bovine and ovine FSH terminate with either sialic acid or sulphate moieties (Green and Baenziger, 1988). In addition multiple FSH isoforms are found both in the pituitary and circulation. This heterogeneity has been ascribed mainly to variations in the degree of glycosylation (Chappel et al., 1983; Baenziger and Green, 1988) but changes in tertiary structure may also be important. It is not known whether the oligosaccharides on the $\alpha$ subunit or $\beta$ subunit are critical for function.

The oligosaccharides on gonadotropins, as in other glycoproteins (Rademacher et al., 1988), play an important role in stabilization, protein confirmation, metabolic half-life and signal transduction (Sairam, 1989). Chemically deglycosylated gonadotropins, while retaining full or enhanced receptor binding ability, have reduced ability to stimulate adenylate cyclase (Sairam and Schiller, 1979; Sairam and Manjunath, 1982; Kalyan and Bahl, 1983; Sairam and Bhargavi, 1985). In addition, chemically deglycosylated gonadotropins (FSH, hCG and LH) antagonize the induction of cyclic adenosine monophosphate (cAMP) by their respective native counterparts in specific target cells (Sairam and Manjunath, 1982; Keutmann et al., 1983; Sairam and Bhargavi, 1985). Although both the $\alpha$ and $\beta$ subunits carry $N$-linked oligosaccharides, glycosylation of the common $\alpha$ subunit (Sairam and Bhargavi, 1985) and in particular the first site from the $\mathrm{N}$-terminus in the $\alpha$ subunit (Matzuk et al., 1989) is consid ered to be critical for adenylate cyclase activation.

Gonadotropins regulate target cell function by binding to surface receptors, activating adenylate cyclase and leading to increased cAMP production (Hunzicker-Dunn and Birnbaumer, 1976). Because FSH stimulates cAMP production and estradiol biosynthesis in Sertoli cells and cAMP stimulates estradiol biosynthesis in Sertoli cells, it is widely accepted that FSH-induced estradiol production is mediated via cAMP (Fritz, 1978; Means et al., 1980). Therefore we tested two hypotheses: (1) that the effects of different oFSH variants on CAMP production and aromatase induction (as measured by estradiol production) would be in parallel; and (2) that deglycosylated 
ovine FSH (DG-oFSH) by virtue of its reported antagonistic ability on native oFSH-induced cAMP production would antagonize the ability of intact oFSH to stimulate aromatase induction in immature rat (7- to 10 -day-old) Sertoli cells. The oFSH variants tested were native oFSH, DGoFSH, asialo oFSH (AS-oFSH), a recombinant of intact $\mathrm{LH} \alpha$ and $\operatorname{FSH} \beta(\alpha+\beta)$ and a recombinant of deglycusylated $\mathrm{LH} \alpha$ and intact $\mathrm{FSH} \beta$ $(\mathrm{DG} \alpha+\beta)$.

\section{Materials and methods}

\section{Materials}

All of the chemicals used were reagent grade. Collagenase (type II) was obtained from Worthington Biochemicals (Freehold, NJ, U.S.A.). Culture supplies were obtained from Gibco (Grand Island, NY, U.S.A.). Neuraminidase was obtained from Sigma (St. Louis, MO, U.S.A.). The native oFSH, DG-oFSH and the subunits used for recombinants were from the same batches of materials used in an earlier study (Sairam and Bhargavi, 1985).

Purified ovine FSH (native oFSH) was isolated from frozen ovine pituitary glands (Sairam, 1979; Manjunath et al., 1982). DG-oFSH was produced by treating oFSH with anhydrous hydrogen fluoride (HF; Manjunath and Sairam, 1982). Deglycosylation resulted in an overall loss of $83 \%$ of the carbohydrate residues, with $86 \%$ loss of neutral hexoses, complete loss of Fuc, NeuAc, and GalNAc, and $56 \%$ loss of GlcNAc. Biochemical studies revealed no detectable changes in the polypeptide backbone (Manjunath et al., 1982). The preparation contained $4.1 \mathrm{~g}$ of carbohydrate per $100 \mathrm{~g}$ of polypeptide (compared to $23.7 \mathrm{~g}$ in the native hormone). Asialo oFSH was prepared by exposing native oFSH to neuraminidase as described by Manjunath and Sairam (1982). Intact $\beta$ subunit (with the full complement of carbohydrate) was obtained by dissociation of native oFSH. Intact $\alpha$ and DG $\alpha$ subunits were prepared from oLH because they were more active than oFSH $\alpha$ subunit in the recombinants, suggesting that the oFSH $\alpha$ subunit may have been slightly altered during isolation (Manjunath and Sairam, 1982; Sairam and Bhargavi, 1985). The oLH $\alpha$ subunit is structurally similar to the oFSH $\alpha$ sub- unit. The $\alpha, \mathrm{DG} \alpha$, and $\beta$ subunits were each dissolved in $0.1 \mathrm{M}$ phosphate buffer $(1 \mathrm{mg} / \mathrm{ml}$; $\mathrm{pH} 7.5$ ) and recombinations were achieved by overnight incubation at room temperature with equal volumes of the $\alpha$ (or DG $\alpha$ ) and $\beta$ subunit solutions. The recombinants that were prepared were intact oLH $\alpha$ with intact oFSH $\beta$ (referred to as the $\alpha+\beta$ recombinant) and DG-oLH $\alpha$ with intact $\mathrm{FSH} \beta$ (referred to as the $\mathrm{DG} \alpha+\beta$ recombinant). Scarcity of highly purified oFSH $\beta$ subunit precluded deglycosylation studies on this material. Therefore, the recombinant of intact $\alpha$ and DG-FSH $\beta$ could not be prepared for simultaneous evaluation.

\section{Immunoassay comparison}

The displacement of ${ }^{125} \mathrm{I}-\mathrm{oFSH}$ by native oFSH, AS-oFSH and DG-oFSH was compared using a modification (Landefeld et al., 1989) of a previously described oFSH radioimmunoassay (Goodman et al., 1981).

\section{Cyclic AMP production}

Immature rat Sertoli cells were prepared and cultured as described for the aromatase induction assay (Padmanabhan et al., 1987, 1989). Following $72 \mathrm{~h}$ of incubation, fresh medium was added and the cultures were treated with varying concentrations of native oFSH, DG-oFSH or the recombinants. Six culture wells were used for each treatment and the experiment was repeated, After $30 \mathrm{~min}$ at $37^{\circ} \mathrm{C}$, the medium was aspirated from three wells of each treatment and stored frozen for CAMP measurement (the other three wells were incubated for $24 \mathrm{~h}$ at $37^{\circ} \mathrm{C}$ to determine aromatase induction). For the determination of intracellular cAMP, the culture plates were frozen at $-70^{\circ} \mathrm{C}$ for $30 \mathrm{~min}$ to rupture the cells. $1 \mathrm{ml}$ of ice-cold $5 \%$ trichloroacetic acid was added to each well and the cells were homogenized by repeated passage through a Pasteur pipette. The homogenate was centrifuged at $4{ }^{\circ} \mathrm{C}$ at $4000 \times g$ for $15 \mathrm{~min}$ and the supernatant extracted with acidified ether. The aqueous phase was dried down and the residue dissolved in 1.0 $\mathrm{ml}$ of Tris-EDTA buffer. Intracellular and extracellular cAMP concentrations were measured in duplicate using a cAMP radioimmunoassay kit from Diagnostic Research Products (DPC; Los 
Angeles, CA, U.S.A.). This assay is reported to have a low cross-reactivity with cGMP $(0.1 \%)$. The cAMP assay sensitivity and half maximal response $\left(E D_{50}\right)$ of the displacement curves were $0.05 \pm 0.01$ and $1.13 \pm 0.19 \mathrm{pmol} /$ tube. The intraassay coefficients of variation at the 80 and $20 \%$ displacement points and the median variance ratio (assay precision) were $7.4 \pm 1.6 \%, 3.7$ $\pm 0.8 \%$, and $0.02 \pm 0.01$, respectively.

\section{Aromatase induction}

The oFSH variants and subunits were compared for their ability to induce aromatase activity in immature rat Sertoli cell cultures using a previously validated Sertoli cell FSH bioassay (Padmanabhan et al., 1987, 1989). The assay measures the FSH stimulated aromatase conversion of $19-\mathrm{OH}$ androstenedione to estradiol $\left(\mathrm{E}_{2}\right)$ in the presence of growth factors and $0.1 \mathrm{mM}$ methyl isobutyl xanthine during a $24 \mathrm{~h}$ incubation with the hormone. Medium $\mathrm{E}_{2}$ concentrations were assayed in duplicate by a previously validated radioimmunoassay (England et al., 1960). The sensitivity (the minimal effective dose) of the $E$, assay was $10 \mathrm{pg} / \mathrm{ml}$, and intra- and interassay coefficients of variation based on a medium pool were less than $8 \%$.

\section{Statistical analyses}

Immunoassays were computed using a computerized program (Duddleson et al., 1970). Potencies of the oFSH variants (comparative dose required for effect) were estimated at the $E_{50}$ points of the various assays. For the bioassay, the dose-response curve for each oFSH variant was fitted by a logistic regression curve using the geometric mean of the zero value as the lower bound and the geometric mean of the highest value as the upper bound. The logistic curve was fitted twice, once using the original data and the second time reusing the data, but weighting each point by the inverse of the height of the curve. Since the variances increased as the expected value increased, the latter is the appropriate analysis and the unweighted analysis was used to compute whether the logistic curve was a good fit to the data. For determining differences between the slopes and $\mathrm{ED}_{50}$ (half maximal increase in estradiol production) of the oFSH variant responses, a test of the difference from the standard (native oFSH) was used as a measure of significance. The cAMP data set was analyzed by analysis of variance after logarithmic transformation of the data. Differences between means were tested by Scheffe's test statistic.

\section{Results}

Comparison of immunological characteristics of the FSH variants

The displacement curves of native oFSH, DGoFSH and AS-oFSH in the oFSH radioimmunoassay are presented in Fig. 1. All three preparations displaced iodinated oFSH to the same degree. The slopes of the log-logit displacement curves for native oFSH, DG-oFSH and AS-oFSH were $-1.05 \pm 0.12,-1.26 \pm 0.06$ and $-1.13 \pm 0.09(n=2)$, respectively. The $\mathrm{ED}_{50}$ points of the displacement curves were $0.91 \pm$ $0.36,0.89 \pm 0.23$ and $0.65 \pm 0.23 \mathrm{ng}$, respectively. The slopes and $\mathrm{ED}_{50}$ points were not significantly different between the three preparations.

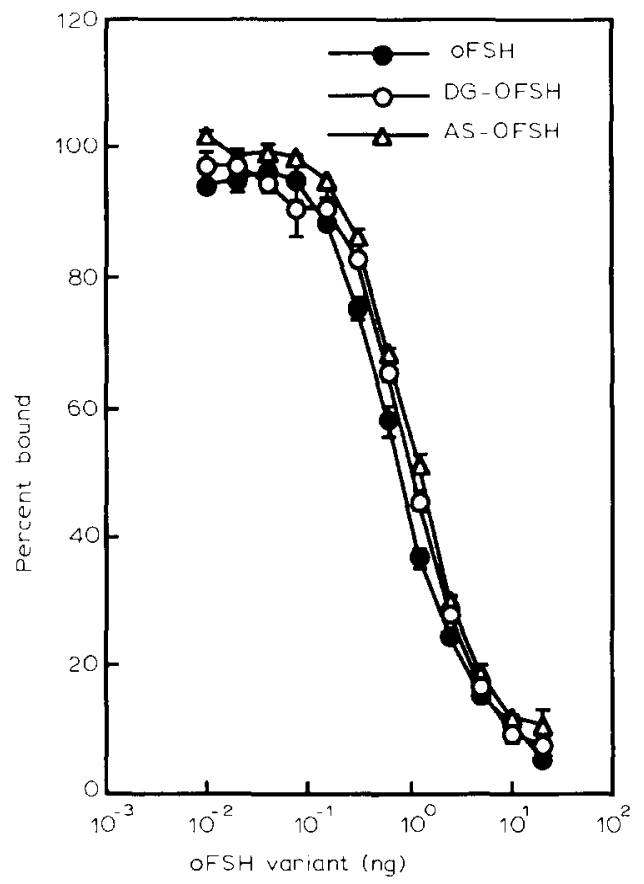

Fig. 1. Competitive displacement curves of ${ }^{125}$ I-labelled oFSH by native oFSH, DG-oFSH and AS-oFSH. 
Comparison of the effect of native oFSH, DG-oFSH and the recombinants on $c A M P$ production from immature rat Sertoli cells

The effects of increasing concentrations of native oFSH, DG-oFSH and the recombinants on their ability to induce cAMP accumulation are summarized in Fig. 2. Extracellular cAMP accumulation (top panel) was increased $(p<0.05)$ by the higher doses (10 ng and higher) of native oFSH, $\alpha+\beta$, and DG $\alpha+\beta$. DG-oFSH $(1-100$ $\mathrm{ng}$ ) had no effect on extracellular cAMP accumulation. The native oFSH and the $\alpha+\beta$ recombinant were equipotent, but a 10 -fold greater amount of the $\mathrm{DG} \alpha+\beta$ recombinant was re-
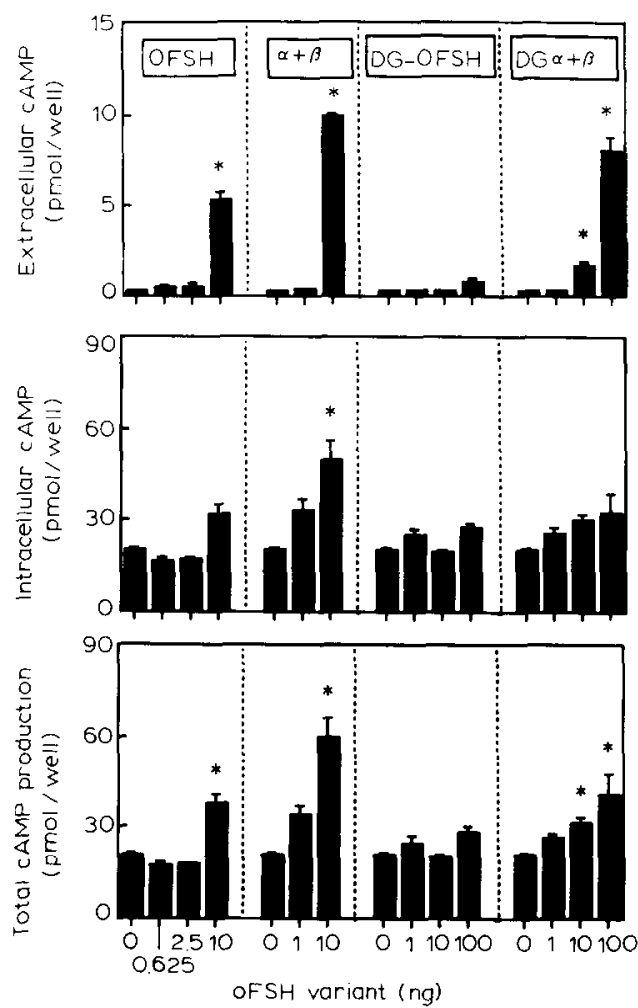

Fig. 2. Effect of graded doses (expressed in $\mathrm{ng}$ ) of native oFSH, DG-oFSH and the $\alpha+\beta$ and DG $\alpha+\beta$ recombinants on extracellular (upper panel), intracellular (middle panel) and total (extracellulart intracellular; lower panel) CAMP accumulation. Values represent mean $\pm \mathrm{SE}$ of three replicates of a representative experiment (one of two). Asterisks indicate significant differences from control cultures (no oFSH). Note the differences in scale between extra- and intracellular (as well as total) cAMP concentrations. For clarity of comparison, basal (no oFSH) cAMP concentrations are presented repeatedly in each panel for each variant oFSH tested. quired to elicit a comparable cAMP response. Intraccllular cAMP (middlc panel) was not significantly increased by the oFSH variants except at high doses of the $\alpha+\beta$ recombinant (10 and 100 ng (not shown)). Total cAMP production (extracellular + intracellular; bottom panel) paralleled extracellular cAMP accumulation.

Comparison of the potency of native oFSH and DG-oFSH in aromatase induction from immature rat Sertoli cells

The dose-response curves of native oFSH and DG-oFSH on aromatase induction are shown in Fig. 3. Increasing concentrations (0.01-1.25 $\mathrm{ng} / \mathrm{ml}$ ) of native oFSH increased $\mathrm{E}_{2}$ production in a linear dose-related manner $(p<0.0001)$. DG-oFSH was less potent in inducing aromatase. As compared to native oFSH 1 , a 16-fold greater concentration of DG-oFSH $\quad(0.025 \pm 0.01$ vs. $0.413 \pm 0.06 \mathrm{ng}$ for native oFSH; mean of seven assays) was required to elicit a minimal detectable increase in $E_{2}$ production. The $E_{50}$ of DG-oFSH was 10 -fold greater than that of native oFSH $(0.33 \pm 0.05$ and $3.27 \pm 1.7 \mathrm{ng}$, respectively; $p<0.01$ ). The relative potency of DG-oFSH as compared to native ofSH was 0.1. Taking allowance for the reduction in molecular weight after deglycosylation would further reduce the relative potency. Although DG-oFSH had reduced potency, it elicited the same maximum $\mathrm{E}_{2}$ response as native oFSH.

\section{Effect of DG-oFSH on oFSH-induced aromatase activity}

The oFSH dose-response curves for aromatase induction in cultures treated with both native and DG-oFSH are shown in Fig. 4. DGoFSH did not inhibit the ability of native oFSH to induce aromatase. The effects of native and DGoFSH were additive at the higher doses of DGoFSH $\left(10^{-1}, 10^{0}, 10^{1}, 10^{2} \mathrm{ng}\right)$. The maximum $\mathrm{E}_{2}$ production was not changed. DG-oFSH, therefore, did not antagonize native oFSH action.

The reduced potency of DG-oFSH is not due to removal of sialic acid

The dose-response curves for aromatase induction by native and AS-oFSH are shown in Fig. 5. AS-oFSH was as potent as native oFSH in 


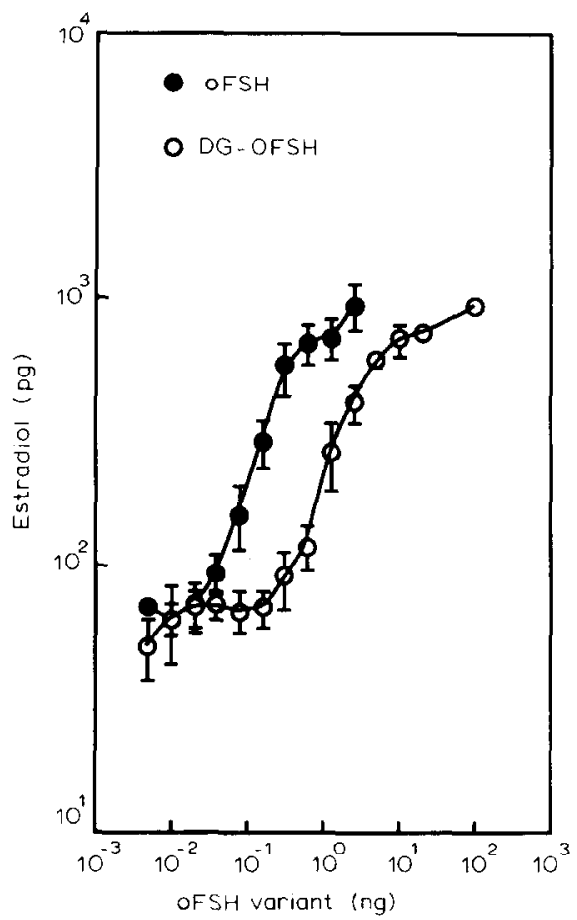

Fig. 3. Effect of deglycosylation of native FSH on aromatase induction. Aromatase induction is measured by the amount of $\mathrm{E}_{2}$ produced. Basal estradiol (no $\mathrm{FSH}$ ) production averaged $53 \pm 8 \mathrm{pg} / \mathrm{ml}$. Values represent mean $\pm \mathrm{SE}$ of seven separate experiments.

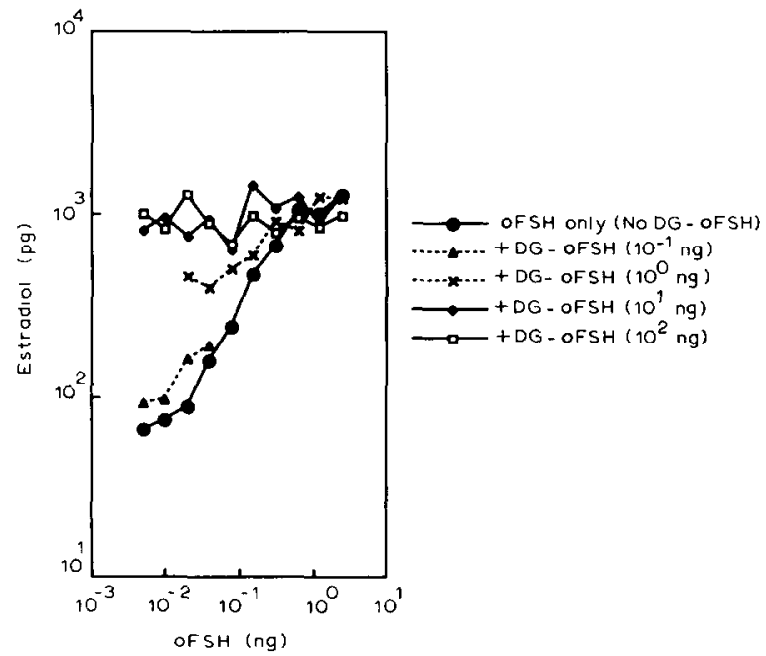

Fig. 4. Effect of DG-oFSH on native oFSH-induced aromatase. Each curve represents responsc to a graded dose of oFSH in the concurrent presence of a particular dose of DG-oFSH. Basal estradiol (no FSH) production averaged $56 \pm 2 \mathrm{pg} / \mathrm{ml}$. Values represent mean of three replicates of a representative experiment (one of two).

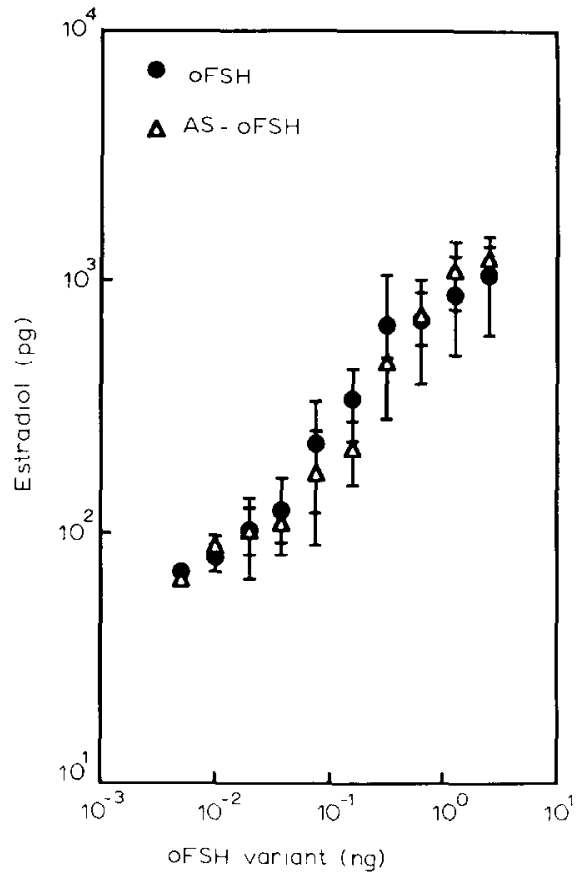

Fig. 5. Effect of AS-oFSH and native oFSH on $\mathrm{E}_{2}$ production by immature Scrtoli cells. Basal cstradiol (no FSH) production averaged $82 \pm 2 \mathrm{pg} / \mathrm{ml}$. Values represent mean $\pm S E$ of three replicates of a representative experiment (one of two).

aromatase induction. The minimum effective doses for aromatase induction for native and AS-oFSH (mean of two replicate experiments) were $0.039 \pm 0.015$ and $0.015 \pm 0.005 \mathrm{ng}$, respectively. The $\mathrm{ED}_{50}$ of the response curves were $0.59 \pm 0.04$ and $0.37 \pm 0.09 \mathrm{ng}$ for native and ASoFSH, respectively $(p>0.1)$. The slopes of the response curves were $1.27 \pm 0.24$ and $1.54 \pm 0.21$ for native and AS-oFSH and were not different. Thus, AS-oFSH is a full agonist of oFSH in aromatase induction. The potency of the native oFSH used for these studies was similar to that of NHPP oFSH-1 (data not shown).

Oligosaccharides residing on the $\alpha$ subunit are not responsible for the reduced potency of DG-oFSH

The oFSH recombinants and subunits were tested for their ability to induce aromatase (Fig. 6). The $\alpha+\beta$ and the $\mathrm{DG} \alpha+\beta$ recombinants were equipotent and increased $\mathrm{E}_{2}$ production in a dose-related manner $(p<0.001)$, indicating that the carbohydrates on the $\alpha$ subunit are not critical for full activity of the hormone. Identical 


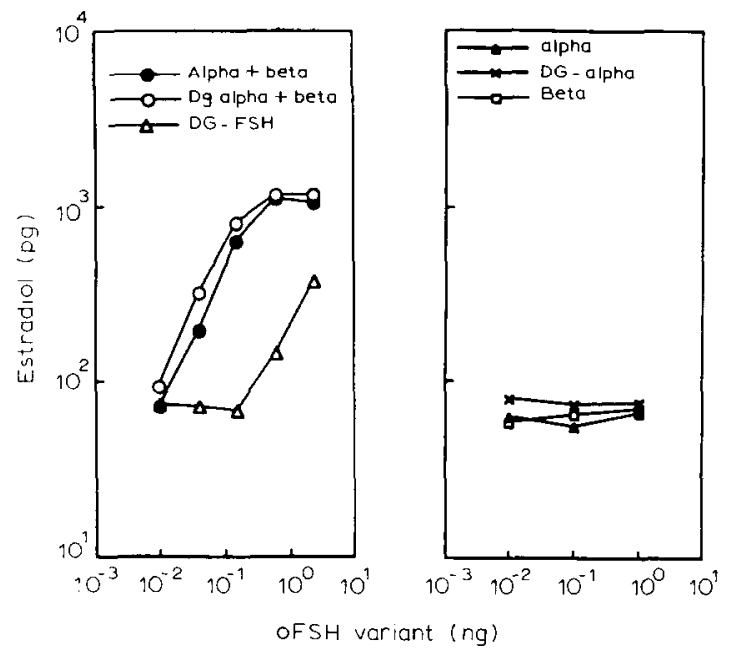

Fig. 6. Effect of $\mathrm{LH} \alpha$, DG $\alpha$, FSH $\beta$, DG-oFSH, $\alpha+\beta$ recombinant (both glycosylated) and $\mathrm{DG} \alpha+\beta$ recombinant on $\mathrm{E}_{2}$ production by immature rat Sertoli cells. Representative responses (one of three experiments) of FSH variants (DGoFSH, $\alpha+\beta$ and $\mathrm{DG} \alpha+\beta$ recombinants) are shown in the left panel and the subunits ( $\mathrm{LH} \alpha, \mathrm{DG} \alpha, \mathrm{FSH} \beta$ ) in the right panel. Basal estradiol (no FSH) production averaged $61 \pm 2$ $\mathrm{pg} / \mathrm{ml}$.

results were obtained during the cAMP experiment, where cAMP and aromatase induction was measured in companion wells (see Fig. 1, aromatase data not shown). The individual subunits (intact $\mathrm{oLH} \alpha$, intact $\mathrm{oFSH} \beta$, and $\mathrm{DG}-\mathrm{oLH} \alpha$ ) had no effect on aromatase induction (Fig. 6).

\section{Discussion}

The results demonstrate that DG-oFSH induces aromatase in immature rat Sertoli cells to the same maximal degree as native oFSH (equivalent efficacy), but with a 10 -fold reduction in potency. DG-oFSH produced an additive response to native oFSH in coincubation. Thus, DG-oFSH is a weak agonist, and not an antagonist, of oFSH-mediated aromatase induction. The finding that AS-oFSH has equivalent immunoand biopotency to the native hormone is in agreement with other reports (Manjunath et al., 1982). Since AS-oFSH is fully potent in induction of aromatase, it appears that loss of carbohydrate moieties other than sialic acid is required to cause a decrease in potency of aromatase induction. A reduction in glycosylation of the $\alpha$ sub- unit cannot account for the reduced potency of DG-oFSH in aromatase induction because selective depletion of oligosaccharides from only the $\alpha$ subunit (DG $\alpha+\beta$ recombinant) did not alter the ability of the recombinant to induce aromatase (Fig. 4). These findings indirectly suggest that the reduced potency of DG-oFSH to induce aromatase may be associated with the loss of oligosaccharides present on the $\beta$ subunit of the hormone. We were unable to test this possibility due to unavailability of DG-FSH $\beta$ subunit. A much greater dose of oFSH was required to cause a significant increase in cAMP than to increase aromatase activity. DG-oFSH, which had a weak agonistic activity for aromatase induction, did not significantly increase cAMP production even at doses as high as $100 \mathrm{ng}$, a concentration 33-fold greater than the $\mathrm{ED}_{50}$ for DG-oFSH induction of aromatase. The $\mathrm{DG} \alpha+\beta$ recombinant increased cAMP production but with a 10 -fold lower potency than the intact $\alpha+\beta$ recombinant. The enhanced ability to produce this second messenger may account for the full steroidogenic (aromatase induction) potential of the $\mathrm{DG} \alpha+\beta$ recombinant.

Despite increasing evidence implicating a role for gonadotropin oligosaccharides in signal transduction, details of the mechanisms of hormone action are not completely defined. Chemically deglycosylated LH, FSH and hCG bind effectively to their specific receptors, often with greater avidity than the native hormones, but are unable to activate adenylate cyclase in their target cells (Sairam and Schiller, 1979; Chen et al., 1982; Manjunath and Sairam, 1982; Manjunath et al., 1982; Kalyan and Bahl, 1983; Keutmann et al., 1983 ) or in non-target cells transfected with receptor cDNA (D. Segaloff, personal communication to M.R.S.). Deglycosylated hCG antagonizes the hCG-mediated induction of adenylate cyclase (Kalyan and Bahl, 1983). Similarly, DG-oFSH antagonizes oFSH induction of adenylate cyclase (Manjunath and Sairam, 1982; Sairam and Bhargavi, 1985), and DG-oLH antagonizes oLH induction of adenylate cyclase (Sairam and Bhargavi, 1985). Using recombinants of deglycosylated subunits, some investigators have reported that adenylate cyclase activation is very sensitive to the loss of carbohydrates on either the $\mathrm{hCG} \alpha$ or 
$\beta$ subunit (Kaylan and Bahl, 1983; Keutmann et al., 1983). Others have reported that the oLHmediated induction of cAMP accumulation was more dependent upon the carbohydrate of the $\alpha$ than the $\beta$ subunit (Sairam and Bhargavi, 1985). By site-directed mutagenesis the first N-glycosylation site in the hCG $\alpha$ has been shown to be very important for function (Matzuk et al., 1989). An oFSH recombinant of deglycosylated oLH $\alpha$ subunit recombined with an intact oFSH $\beta$ subunit lacked the capacity to induce adenylate cyclase (Sairam and Bhargavi, 1985). In this study, we have shown that the $\mathrm{DG} \alpha+\beta$ recombinant increased extracellular cAMP accumulation (as well as total production) albeit to a lesser degree than the native hormone and the $\alpha+\beta$ recombinant (Fig. 2). In contrast, DG-oFSH did not cause a similar increase in cAMP accumulation. These results differ from previously published data (Sairam and Bhargavi, 1985) in which identical recombinants were used. This discrepancy may be related to the fact that our studies were performed with isolated young rat Sertoli cells from 7- to 10-day-old rats in culture, while the previous studies were done with seminiferous tubules from 19-day-old rats. In addition, we have used a more sensitive cAMP assay, and have segregated the extracellular and intracellular responses.

The complete dependency of the cAMP response upon the presence of intact oligosaccharides in gonadotropins is in contrast to the effect of deglycosylation on the induction of steroidogenesis. Chemical deglycosylation of native oLH results in near complete loss of steroidogenic activity (Sairam and Schiller, 1979). Recombination studies have revealed that $\mathrm{DG} \alpha$-intact $\mathrm{LH} \beta$ has only $35 \%$ of the steroidogenic capacity of the native hormone, but intact $\alpha$-DGLH $\beta$ has full steroidogenic activity (Sairam, 1980). Similar results were also reported for deglycosylated oLH by Liu et al. (1984). Similarly, the steroidogenic capacity of hCG is more dependent on the $\alpha$ subunit oligosaccharides (Goverman et al., 1982). In addition, both these deglycosylated hormones (oLH and hCG) antagonize the steroidogenic activity of their native hormones (Sairam and Schiller, 1979; Kalyan and Bahl, 1983; Liu et al., 1984). The effect of deglycosylation of FSH on the steroidogenic response has not been previ- ously reported. Our data suggests that the effect of deglycosylation of the $\alpha$ subunit on the steroidogenic inductive potency of oFSH is different from that seen with the other two gonadotropins ( $\mathrm{LH}, \mathrm{hCG}$ ).

The dichotomy observed between adenylate cyclase activation and aromatase induction is intriguing, because cAMP is believed to be a second messenger for FSH signal transduction (Fritz, 1978; Means et al., 1980). After binding to the receptor, FSH increases target cell cAMP by stimulating adenylate cyclase (Braun, 1974; Abou-lssa and Reichert, 1979) and decreasing phosphodiesterase activity (Means et al., 1980; Conti et al., 1983). In our study and others, aromatase was induced at FSH concentrations that were much smaller than those required to elicit detectable increases in cAMP levels (Moyle and Ramachandran, 1973). The available intracellular cAMP may be sufficient to elicit the maximal steroidogenic response or the functional pool of cAMP may be compartmentalized and immeasurable (Albertson and Vaitukaitis, 1977). In rat Leydig cells, occupancy of only $15-20 \%$ of the intracellular cAMP receptors is associated with a maximum steroidogenic response (Dufau et al., 1977, 1978) and total intracellular cAMP levels are 10 -fold greater than receptor protein bound cAMP levels (Schumacher et al., 1979). An alternative explanation for this discrepancy is that oFSH induction of aromatase may be mediated through another signal transduction pathway. It has been suggested that some of the FSH effects in Sertoli cells are mediated by changes in intracellular $\mathrm{Ca}^{2+}$ (Means et al., 1980). No direct evidence for $\mathrm{Ca}^{2+}$ mediation of $\mathrm{FSH}$ action in Sertoli cells is yet available.

Although the reduced potency of the deglycosylated hormone is attributed to the loss of carbohydrates, subtle secondary conformational changes may have occurred in the polypeptide backbone (Keutmann et al., 1985; Sairam et al., 1988) despite the lack of detectable change in conformation of deglycosylated hormones in biophysical studies (Manjunath and Sairam, 1982; Manjunath et al., 1982). Confirmation that subtle changes may occur is provided by the generation of antisera that specifically recognize deglycosylated but not native hormones (Sairam et al., 
1988; Lamarre and Sairam, 1989). Using a polyclonal antibody, we did not find any difference in the antigenic properties of the native and deglycosylated hormones.

We conclude that: (1) the effects of oFSH variants on cAMP production and aromatase induction are not parallel; (2) $\mathrm{DG}$-oFSH is a weak agonist (and not an antagonist) of oFSH in aromatasc induction; (3) the reduced potency of DG-oFSH must involve oligosaccharides internal to sialic acid; and (4) the carbohydrate moiety on $\mathrm{FSH} \beta$ may be essential for full expression of aromatase induction. Since DG-oFSH does not produce detectable increases in cAMP accumulation, but is able to induce aromatase, the FSHmediated induction of aromatase may involve other signal transduction pathways. Alternatively, the maximal steroidogenic response may be induced by small, but undetectable, increases in cAMP.

\section{Acknowledgements}

We are grateful to Ms. Joanne Sonstein, Maria Barondy, and Katherine Kersey for expert technical assistance, the Standards and Reagents Core Facility of the Reproductive Sciences Program for radioimmunoassay reagents, and Dr. Gordon D. Niswender (Colorado Statc Univcrsity) for RIA reagents. Finally we are grateful to the National Hormone and Pituitary Program for the purified oFSH.

\section{References}

Abou-Issa, H. and Reichert, Jr., L.E. (1979) Endocrinology 104, 189-193.

Albertson, B.D. and Vaitukaitis, J.D. (1977) Endocrinology 101, 1413-1420.

Baenziger, J.U. and Green, E.D. (1988) Biochim. Biophys. Acta $947,287-306$.

Braun, T. (1984) in Hormone Binding and Target Cell Activation in the Testis (Dufau, M.L. and Means, A.R., eds.), pp. 243-264, Plenum Press, New York.
Chappel, S.C., Ulloa-Aguirre, A. and Coutifaris, C. (1983) Endocr. Rev. 4, 179-211.

Chen, H.-C., Shimohigashi, Y., Dufau, M.L. and Catt, K.J. (1982) J. Biol. Chem. 257, 14446-14452.

Conti, M., Toscano, M.V., Petrelli, L., Geremia, R. and Stefanini, M. (1983) Endocrinology 113, 1845-1853.

Duddleson, W.G., Midgley, Jr., A.R. and Niswender, G.D. (1972) Cumput. Biomed. Res. 5, 205-210.

Dufau, M.L., Horner, K.A., Hayashi, K., Tsuruhara, T., Conn, P.M. and Catt, K.J. (1978a) J. Biol. Chem. 253, 3721-3729.

Dufau, M.L., Tsuruhara, T., Horner, K.A., Podesta, E.J. and Catt, K.J. (1978b) Proc. Natl. Acad. Sci. U.S.A. 74, 34193423.

England, B.G., Niswender, G.D. and Midgley, Jr., A.R. (1974) J. Clin. Endocrinol. Metab. 38, 42-50.

Fritz, I.G. (1978) in Biochemical Actions of Hormones, Vol. 5 (Litwach, G., ed.), pp. 249-281, Academic Press, New York.

Goodman, R.L., Pickover, S.M. and Karsch, F.J. (1981) Endocrinology 108, 772-777.

Goverman, J.M., Parsons, T.F. and Pierce, J.G. (1982) J. Biol. Chem. 257, 15059-15064.

Green, E.D. and Baenziger, J.U. (1988) J. Biol. Chem. 263, 25-35.

Hunzicker-Dunn, M. and Birnbaumer, L. (1976) in Luteinizing Hormone Action and Receptors (Ascoli, M., ed.), pp. 57-135, CRC Press, Boca Raton, FL.

Kalyan, N.K. and Bahl, O.P. (1983) J. Biol. Chem. 258, 67-74.

Keutmann, H.T., Mcllroy, D.J., Bergert, E.R. and Ryan, R.L. (1983) Biochemistry 22, 3067-3072.

Keutmann, H.T., Johnson, L. and Ryan, R.J. (1985) FEBS Lett. 185, 333-338.

Lamarre, L. and Sairam, M.R. (1989) Mol. Cell. Endocrinol. 66, 181-187.

Landefeld, T.D., Ebling, F.J.P., Suttie, J.M., Vannerson, L.A., Padmanabhan, V., Beitins, I.Z. and Foster, D.L. (1989) Endocrinology 125, 351-356.

Liu, W.-K., Young, J.D. and Ward, D.N. (1984) Mol. Cell. Endocrinol. 37, 29-39.

Manjunath, P. and Sairam, M.R. (1982) J. Biol. Chem. 257, 7109-7115.

Manjunath, P., Sairam, M.R. and Sairam, J. (1982) Mol. Cell. Endocrinol. 28, 125-138.

Matzuk, M.M., Keene, J.L. and Boime, I. (1989) J. Biol. Chem. 264, 2409-2414.

Means, A.R., Dedman, J.R., Tash, J.S., Tindall, D.J., VanSickle, M. and Welsh, M.J. (1980) Annu. Rev. Physiol. 42, 59-70.

Moyle, W.R. and Ramachandran, J. (1973) Endocrinology 93 , 127-134.

Padmanabhan, V., Chappel, S.C. and Beitins, I.Z. (1987) Endocrinology 121, 1089-1098.

Padmanabhan, V., Ebling, F.J.P., Sonstein, J., Fenner, D.E., Kelch, R.P., Foster, D.L. and Beitins, I.Z. (1989) Endocrinology 125, 2517-2526.

Rademacher, T.W., Parekh, R.B. and Dwek, R.A. (1988) Annu. Rev. Biochem. 57, 785-838.

Sairam, M.R. (1979) Arch. Biochem. Biophys. 194, 71-78. 
Sairam, M.R. (1980) Arch. Biochim. Biophys, 204, 199-206. Sairam, M.R. (1983) in Hormonal Proteins and Peptides, Vol. 11 (Li, C.H., ed.), pp. 1-79, Academic Press, New York.

Sairam, M.R. (1989) FASEB J. 3, 1915-1926.

Sairam, M.R. and Bhargavi, G.N. (1985) Science 229, 65-67.

Sairam, M.R. and Manjunath, P. (1982) Mol. Cell. Endocrinol. 28, 139-150.
Sairam, M.R. and Schiller, P.W. (1979) Arch. Biochem. Biophys. 197, 294-301.

Sairam, M.R., Linggen, J. and Bhargavi, G.N. (1988) Biosci. Rep. 8, 271-278.

Schumacher, M., Schafer, G., Lichtenberg, V. and Hilz, H. (1979) FEBS Lett. 107, 398-402. 